\title{
The Effect of Eight Weeks of Testosterone Enanthate Consumption on Oxidative Indicators of Kidney Tissue in Resistance Trained Rats
}

\author{
Masoumeh Mehrabi $^{\circledR}$, Yaser Kazemzadeh ${ }^{1 *}$, Ali Gorzi $^{2}$, Seyed Ali Hosseini ${ }^{3}$, Saeid Sedaghati ${ }^{4}$ \\ ${ }^{1}$ Department of Exercise Physiology, Islamshahr Branch, Islamic Azad University, Islamshahr, Iran \\ ${ }^{2}$ Department of Sport Sciences, University of Zanjan, Zanjan, Iran \\ ${ }^{3}$ Department of Exercise Physiology, Marvdasht Branch, Islamic Azad University, Marvdasht, Iran \\ ${ }^{4}$ Department of Sport Management, Islamshahr Branch, Islamic Azad University, Islamshahr, Iran
}

\section{*Correspondence to \\ Yaser Kazemzadeh, \\ Tel: +989122205973, \\ Email: yaser.kazemzadeh@yahoo.com}

Received September 10, 2020 Accepted November 10, 2020 Published online December 30, 2020

\begin{abstract}
Introduction: Taking anabolic steroids can affect the state of oxidative stress in different tissues of the body such as kidneys. This study aimed to examine the effect of 8 weeks of resistance training with testosterone enanthate consumption on tissue oxidation indicators in male rats. Methods: In the current experimental study, 18 rats with an average weight of $250 \pm 50 \mathrm{~g}$ were divided into 3 groups of 6 animals including: (1) control, (2) training, and (3) training + testosterone. Rats in the training and training + testosterone groups underwent resistance training for 8 weeks and 5 sessions per week. Also, rats in the training + testosterone group received $20 \mathrm{mg} / \mathrm{kg}$ of testosterone enanthate by intramuscular injection 3 days a week. The resistance training protocol consisted of climbing a 1-meter ladder 5 days a week (4 sets of 6 with a rest interval of 60 to 90 seconds) in which the weights were $60 \%$ of the rats' body weights in the first week, and $20 \%$ of the body weight was added to the weights every week. One-way analysis of variance was used to analyze the research findings $(P \leq 0.05)$.

Results: Glutathione peroxidase (GPx) level in the training + testosterone group was significantly lower than the control group $(P=0.007)$; however, there were no significant differences in malonaldehyde (MDA) $(P=0.64)$ and superoxide dismutase (SOD) $(P=0.29)$ levels between the research groups.

Conclusion: It seems that testosterone consumption can be effective in increasing oxidative stress factors in the kidney tissue of resistance-trained rats.

Keywords: Oxidative stress, Testosterone enanthate, Resistance training, Kidney.
\end{abstract}

Please cite this article as follows: Mehrabi $M$ Kazemzadeh Y, Gorzi A, Hosseini SA, Sedaghati S. The Effect of eight weeks of testosterone enanthate consumption on oxidative indicators of kidney tissue in resistance trained rats. Int J Basic Sci Med. 2020;5(4):155159. doi: $10.34172 /$ ijbms.2020.27.

\section{Introduction}

Anabolic androgenic steroids (AAS) are testosterone-originated compounds that exhibit androgenic or anabolic effects depending on the target tissue. ${ }^{1}$ Testosterone, often referred to as the father of steroids, was first extracted in 1935 by Buttenet et al, and after that, the use of this hormone without legal prohibition became common for the treatment of specific diseases and for general and specialized strengthening. Its medicinal type, called testosterone enanthate, is a natural androgenic hormone which is widely used by athletes. ${ }^{2}$ It has been reported that $87 \%$ of these performance-enhancing drugs among men and women are testosterone enanthate and testosterone propionate, which are used by $6.4 \%$ of men and $1.6 \%$ of women. ${ }^{3}$ The use of AAS in medicine is limited, and it is only used with great caution in some disorders such as stunted growth, osteoporosis, hypogonadism and anemia, infertility, kidney disease, aplastic anemia, osteoporosis, AIDS and some cancers. AAS drugs are typically employed in higher doses and for longer periods among athletes, e.g., weightlifters, swimmers and bodybuilders to enhance muscle mass, physical function and strength. ${ }^{4}$ Such implementations can lead to side effects on various organs, including kidneys. ${ }^{5,6}$

Kantarci et $\mathrm{al}^{5}$ showed an escalation in renal volume, renal cortical thickness and blood urea nitrogen (BUN) in the groups

(c) 2020 The Author(s); Published by Zabol University of Medical Sciences. This is an open-access article distributed under the terms of the Creative Commons Attribution License (http://creativecommons.org/licenses/by/4.0), which permits unrestricted use, distribution, and reproduction in any medium, provided the original work is properly cited. 
using AAS compared to non-AAS groups. These results were also reported by Frati et al. ${ }^{7}$ AAS abuse can alter renal morphology (as shown by renal hypertrophy) and also increase oxidative stress, and collagen deposition. ${ }^{6,8}$ Oxidative stress is an important parameter that can damage organ, and this metabolic disorder is characterized by either a decline in the antioxidant enzyme system and an increment in reactive oxygen species. ${ }^{9}$ Oxidative stress can be driven by the humoral system controlling blood pressure, especially by the renin-angiotensin system. ${ }^{10}$ Research by de Lima et $\mathrm{al}^{11}$ showed that nandrolone consumption caused renal hypertrophy, which was associated with decreased organ function and increased collagen deposition.

Increased oxidative stress is followed by decreased superoxide dismutase (SOD) activity; also, nandrolone consumption in rats due to increased oxidative stress in renal tissue, leads to renal damage and renal fibrosis. Exercise has been reported to play an important role in the pharmacokinetics of drugs. Exercise and physical activity increase blood circulation in the liver and accelerate biotransformation and excretion of substances by kidneys. ${ }^{12}$ Ahmadizadeh et al showed that regular exercise reduced the toxic effects of morphine on the liver and kidneys. ${ }^{13}$ Since regular exercise can possibly reduce the toxic effects of drugs, the role of exercise in reducing drug side effects is significant. ${ }^{14}$

Research has shown that resistance training can counteract the increase in oxidative stress as a result of increased levels of free radicals. ${ }^{15}$ On the other hand, it has been observed that a training session, depending on its duration and severity, can cause different intensities of oxidative damage. ${ }^{16}$ Some reports suggest that in response to resistance training, free radicals and reactive oxygen species (ROS) are increased during training sessions. ${ }^{17}$ Riezzo et $\mathrm{al}^{18}$ showed an escalation in oxidative stress and a diminution in the ability to inhibit radicals in the kidney tissue of the steroid group along with strenuous exercise. Many athletes use high amounts of steroid use. ${ }^{19}$ There is little research on changes in renal oxidative enzymes due to the use of this steroid along with high-intensity resistance training. However, research has shown improvements in the function of antioxidant defense enzymes and overall improvement in oxidative stress due to resistance training. ${ }^{15}$ Therefore, this study aimed to examine the effect of eight weeks of resistance training alone and in combination with testosterone enanthate consumption on oxidative indicators of the kidney tissue in male rats.

\section{Materials and Methods}

In the present study, 18 eight-week male rats with an average weight of $250 \pm 50 \mathrm{~g}$ were prepared from the Pasteur Institute (Iran) and transferred to the Sports Physiology Laboratory of Zanjan University. Rats were then maintained in a standard environment for 8 days with a 12 -hour light/dark cycle, a temperature of $22 \pm 2^{\circ} \mathrm{C}$, and a humidity of $45 \pm 5 \%$ for adapting to the new conditions. The rats were randomly categorized into three groups, including: (1) control $(n=6),(2)$ training $(n=6)$, and (3) training + testosterone $(n=6)$. Rats in the training and training + testosterone groups underwent resistance training for eight weeks and 5 sessions per week. Also, rats in the third group received $20 \mathrm{mg} / \mathrm{kg}$ of intramuscular testosterone enanthate (manufactured by Iran Hormone Company, Iran, the batch number of 0069) three days a week. ${ }^{20}$

\section{Training Protocol}

In the present study, the resistance training protocol comprised 5 days of training per week (4 sets of 6 with a rest interval of 60 to 90 seconds) in the form of climbing a 1-meter ladder with 26 steps and tying weights to the tails of rats in which weights were $60 \%$ of the rats' body weights in the first week, and $20 \%$ of body weight was added to the weights every week; also, 2 sessions of weighing were carried out per week. ${ }^{21}$

\section{Biochemical Measurements}

Forty-eight hours after the last training session and testosterone consumption, rats were anesthetized with xylazine and ketamine, and kidney tissue was extracted and transferred to the laboratory for measuring the variables. SOD activity was determined using the Randox Laboratories Ltd, UK kit, according to the manufacturer's method.22 Glutathione peroxidase (GPx) activity was evaluated via the Randox Laboratories Ltd kit, the UK, according to Paglia and Valentine methods, ${ }^{23}$ and malonaldehyde (MDA) activity was measured via Mihara and Uchiyama method based on the manufacturer's protocol. $^{24}$

\section{Statistical Analyses}

For analyzing the findings, the Shapiro-Wilk procedure, one-way analysis of variance, and Tukey's post hoc statistical test were employed $(P \leq 0.05)$.

\section{Results}

GPx, MDA and SOD protein levels have been shown in Figures 1, 2, and 3, respectively. The results of the ShapiroWilk test showed that the data distribution of GPx, MDA, and SOD in the three groups of research was normal $(P \leq 0.05)$. The results of one-way ANOVA showed that there was a significant difference in GPx levels between the research groups (Figure 1). However, there was no significant difference in MDA levels (Figure 2) as the $P$ and $F$ values confirmed these results $(P=0.64)$. The SOD levels did not show a significant difference as $P$ and $F$ values (Figure 3, $P=0.29$ ). The results of Tukey's post hoc test showed that GPx levels in the training + testosterone group was significantly lower compared to the control group $(P=0.007)$. 


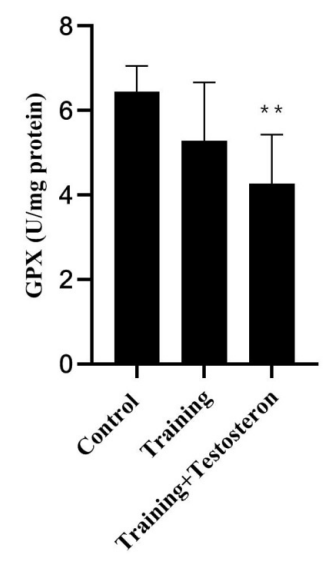

Figure 1. Serum GPx Levels in the Studied Groups. ${ }^{* *} P \leq 0.01$; significant decline as compared to the control group.

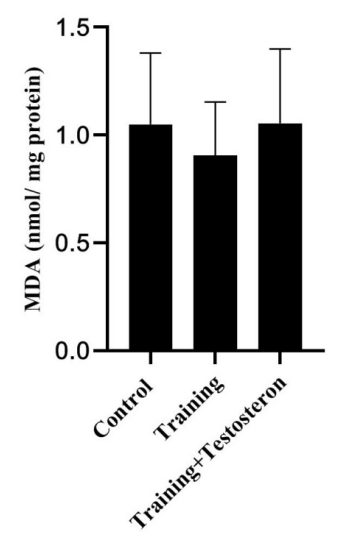

Figure 2. Serum MDA Levels in the Studied Groups.

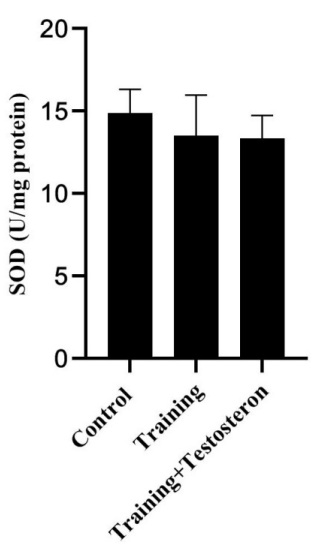

Figure 3. Serum SOD Levels in the Studied Groups.

\section{Discussion}

The results revealed that eight weeks of resistance training had no significant effect on the levels of SOD, MDA and GPx in kidney tissue. It has been reported that high intensity resistance activity can increase oxidative stress and impair cell redox status (oxidationreduction). It has also been exhibited that oxidative stress during higher intensity activity is greater as compared to low-intensity resistance activity. ${ }^{25}$ Although the exact mechanism of ROS production and oxidative stress after resistance training is not known, possible mechanisms responsible for ROS formation due to resistance training could be xanthine oxidase pathway, respiratory burst of neutrophils, catecholamine autooxidation, localized muscle ischemia, conversion of weak superoxide to strong hydroxyl radical by lactic acid and altered calcium homeostasi. ${ }^{26}$ On the other hand, it has been shown that regular and continuous exercise declines lipid and protein peroxidation by enhancing antioxidant defense. ${ }^{1}$

The results of the studies conducted by Venojarvia et $\mathrm{al}^{27}$ and Park \& $\mathrm{Kwak}^{28}$ are similar to the results of the present study. Park and Kwak measured the effects of aerobic and anaerobic training on three groups of endurance athletes, resistance athletes, and non-trained individuals, in which no change in oxidative stress status and antioxidant capacity was observed, which could be attributed to redox/resuscitation balance. ${ }^{28}$ On the other hand, recent findings show that resistance training has different effects on oxidative stress. In contrast to the present findings, Ezabadi et al showed that 4 weeks of resistance training leads to a significant increase in SOD and CAT levels as well as a significant decrease in the cerebral MDA of diazinon-poisoned rats, ${ }^{29}$ which is contradictory with the results of this study. The main reasons for these contradictory results are differences in the antioxidant capacity of the investigated tissue, differences in tissue sampling time, differences in the type of resistance training protocols and intensity and duration of exercise, as well as laboratory methods of measuring the desired concentrations.

The reason for the lack of change in the activity of antioxidant enzymes in this study can be explained by several possible factors. The intensity and duration of the exercise may not have been such that it increased the production of reactive oxygen species in the kidney tissue.

On the other hand, it is possible that normal levels of renal antioxidant enzymes are responsive to the radicals produced following this type of exercise, which may be a good justification for not altering the activity of these enzymes. Regarding interactive effects, the results of the present study showed that GPx levels in the training and testosterone group were significantly lower than the control group but there was no significant difference in MDA and SOD levels between the study groups.

Similar to the results of the present study, Riezzo et $\mathrm{al}^{18}$ showed that the use of anabolic steroids along with high intensity training reduces the enzymatic activity of GR and the antioxidant GPx and thus reduces the ability of the kidneys to inhibit radicals. Also, the findings of this study are consistent with the findings of Kara et $\mathrm{al}^{30}$ who showed that the use of anabolic steroids increases catalase, while the parameters of MDA, GSH, SOD did not change significantly. Research by de Lima et $\mathrm{al}^{11}$ showed that nandrolone consumption increased oxidative stress in both proteins and lipids and subsequently decreased SOD 
activity. Oxidative stress is an important parameter that increases organ damage, and this metabolic disorder is characterized by either an increase in reactive oxygen species or a decrease in the antioxidant enzyme system. ${ }^{9}$ Decreased antioxidant enzymes, due to their high use to reduce ROS, may change the redox status to oxidative stress, ${ }^{31}$ and the decrease in GPx antioxidant activity in this study is probably due to the response to these conditions. Anabolic steroids may have a direct toxic effect on glomerular cells, leading to the accumulation of the mesangial matrix and the depletion of podocytes, which are independent of structural-functional adaptations. ${ }^{28}$ The results of the previous studies have shown that testosterone can cause oxidative stress directly and also indirectly by activating the renin-angiotensin-aldosterone system and endothelin (by regulating NADPH oxidase). ${ }^{30}$

It can be mentioned that the measuring of the variables by western blotting and real-time polymerase chain reaction (PCR) methods, and also studying the effects of resistance training and testosterone consumption on liver and heart tissues can be interesting issues in the future research.

\section{Conclusion}

In our study, there were no significant differences in MDA and SOD levels between the research groups while GPx levels revealed a significant difference in the training + testosterone group in comparison to the control group. In addition, it appears that testosterone consumption can be effective in increasing anti-oxidative stress factors in the kidney tissue of resistance trained rats.

\section{Ethical Approval}

The study was approved by the Ethical Committee of Tehran Islamic Azad University of Medical Sciences (IR. IAU.TMU.REC.1399.347).

\section{Conflict of Interest Disclosure}

The authors have no conflict of interest to declare.

\section{Authors' Contributions}

All authors had equal contribution to the writing and revision of this paper.

\section{Acknowledgements}

The authors express their gratitude and appreciation for the spiritual support of the Islamshahr Branch of Islamic Azad University.

\section{References}

1. Simão VA, Berloffa Belardin L, Araújo Leite GA, de Almeida Chuffa LG, Camargo IC. Effects of different doses of nandrolone decanoate on estrous cycle and ovarian tissue of rats after treatment and recovery periods. Int J Exp Pathol. 2015;96(5):338-349. doi:10.1111/iep.12144

2. Bhasin S, Cunningham GR, Hayes FJ, et al. Testosterone therapy in men with androgen deficiency syndromes: an Endocrine Society clinical practice guideline. J Clin Endocrinol Metab. 2010;95(6):2536-2559. doi:10.1210/ jc.2009-2354

3. Sagoe D, Molde H, Andreassen CS, Torsheim T, Pallesen $\mathrm{S}$. The global epidemiology of anabolic-androgenic steroid use: a meta-analysis and meta-regression analysis. Ann Epidemiol. 2014;24(5):383-398. doi:10.1016/j. annepidem.2014.01.009

4. Silva DK, Esteves A, Da Ré Guerra F, et al. Chronic use of anabolic steroids and the effects on the neuronal density of the cerebral cortex and hippocampus in mice. Am J Sports Sci. 2018;6(3):122-129. doi:10.11648/j.ajss.20180603.18

5. Kantarci UH, Punduk Z, Senarslan O, Dirik A. Evaluation of anabolic steroid induced renal damage with sonography in bodybuilders. J Sports Med Phys Fitness. 2018;58(11):16811687. doi:10.23736/s0022-4707.17.06763-9

6. Tsitsimpikou C, Vasilaki F, Tsarouhas $\mathrm{K}$, et al. Nephrotoxicity in rabbits after long-term nandrolone decanoate administration. Toxicol Lett. 2016;259:21-27. doi:10.1016/j.toxlet.2016.06.1122

7. Frati P, Busardò FP, Cipolloni L, Dominicis ED, Fineschi V. Anabolic Androgenic Steroid (AAS) related deaths: autoptic, histopathological and toxicological findings. Curr Neuropharmacol. 2015;13(1):146-159. doi:10.2174/157015 $9 \times 13666141210225414$

8. Brasil GA, Lima EM, Nascimento AM, et al. Nandrolone decanoate induces cardiac and renal remodeling in female rats, without modification in physiological parameters: the role of ANP system. Life Sci. 2015;137:65-73. doi:10.1016/j. lfs.2015.07.005

9. Ara N, Nakkanong K, Lv W, Yang J, Hu Z, Zhang M. Antioxidant enzymatic activities and gene expression associated with heat tolerance in the stems and roots of two cucurbit species ("Cucurbita maxima" and "Cucurbita moschata") and their interspecific inbred line "Maxchata". Int J Mol Sci. 2013;14(12):24008-24028. doi:10.3390/ ijms141224008

10. Crowley SD, Coffman TM. Recent advances involving the renin-angiotensin system. Exp Cell Res. 2012;318(9):10491056. doi:10.1016/j.yexcr.2012.02.023

11. de Lima EM, dos Santos Cassaro K, da Silva CL, et al. Eight weeks of treatment with nandrolone decanoate in female rats promotes disruption in the redox homeostasis and impaired renal function. Life Sci. 2020;242:117227. doi:10.1016/j.lfs.2019.117227

12. Alishahi A, Nasiri S. The protective effect of regular aerobic training on doxorubicin-induced hepatotoxicity in rats: the insulin-like growth factor system. Zahedan J Res Med Sci. 2014;16(12):59-64. [Persian].

13. Ahmadizadeh M, Sarkaki A, Farboud Y, Mohammadian B, Rahim F. Effect of exercise on morphine-induced toxicity in rat liver and kidney. Jundishapur Sci Med J. 2012;11(3):325-333. [Persian].

14. Guo R, Liong EC, So KF, Fung ML, Tipoe GL. Beneficial mechanisms of aerobic exercise on hepatic lipid metabolism in non-alcoholic fatty liver disease. Hepatobiliary Pancreat Dis Int. 2015;14(2):139-144. doi:10.1016/s14993872(15)60355-1

15. Sallam N, Laher I. Exercise modulates oxidative 
stress and inflammation in aging and cardiovascular diseases. Oxid Med Cell Longev. 2016;2016:7239639. doi:10.1155/2016/7239639

16. Bejma J, Ramires P, Ji LL. Free radical generation and oxidative stress with ageing and exercise: differential effects in the myocardium and liver. Acta Physiol Scand. 2000;169(4):343-351. doi:10.1046/j.1365201x.2000.00745.x

17. Leeuwenburgh C, Heinecke JW. Oxidative stress and antioxidants in exercise. Curr Med Chem. 2001;8(7):829838. doi:10.2174/0929867013372896

18. Riezzo I, Turillazzi E, Bello S, et al. Chronic nandrolone administration promotes oxidative stress, induction of proinflammatory cytokine and TNF- $\alpha$ mediated apoptosis in the kidneys of CD1 treated mice. Toxicol Appl Pharmacol. 2014;280(1):97-106. doi:10.1016/j.taap.2014.06.031

19. Bahari S, Faramarzi M, Azamian Jazi A, Cheragh Cheshm $\mathrm{M}$. The effect of 8 week resistance training on resting level of liver inflammatory markers and insulin resistance of type 2 diabetic women. Armaghane Danesh. 2014;19(5):450561. [Persian].

20. Dornelles GL, Bueno A, de Oliveira JS, et al. Biochemical and oxidative stress markers in the liver and kidneys of rats submitted to different protocols of anabolic steroids. Mol Cell Biochem. 2017;425(1-2):181-189. doi:10.1007/s11010016-2872-1

21. Gorzi A, Rajabi H, Gharakhanloo R, Dehkhoda MR, Hedayati M. The effects of 8 weeks of resistance training on total and A12 acetyl cholinesterase activity in slow twitch muscles of rats. Research in Sport Medicine and Technology. 2017;15(13):9-16. [Persian].

22. Pourmemar E, Majdi A, Haramshahi M, Talebi M, Karimi P, Sadigh-Eteghad S. Intranasal cerebrolysin attenuates learning and memory impairments in D-galactose-induced senescence in mice. Exp Gerontol. 2017;87(Pt A):16-22. doi:10.1016/j.exger.2016.11.011

23. Paglia DE, Valentine WN. Studies on the quantitative and qualitative characterization of erythrocyte glutathione peroxidase. J Lab Clin Med. 1967;70(1):158-169. doi:10.5555/uri:pii:0022214367900765

24. Mihara M, Uchiyama M. Determination of malonaldehyde precursor in tissues by thiobarbituric acid test. Anal Biochem. 1978;86(1):271-278. doi:10.1016/00032697(78)90342-1

25. Güzel NA, Hazar S, Erbas D. Effects of different resistance exercise protocols on nitric oxide, lipid peroxidation and creatine kinase activity in sedentary males. J Sports Sci Med. 2007;6(4):417-422.

26. Arazi H, Mohammadjafari H, Asadi A. Use of anabolic androgenic steroids produces greater oxidative stress responses to resistance exercise in strength-trained men. Toxicol Rep. 2017;4:282-286. doi:10.1016/j. toxrep.2017.05.005

27. Venojärvi M, Korkmaz A, Wasenius $\mathrm{N}$, et al. 12 weeks' aerobic and resistance training without dietary intervention did not influence oxidative stress but aerobic training decreased atherogenic index in middle-aged men with impaired glucose regulation. Food Chem Toxicol. 2013;61:127-135. doi:10.1016/j.fct.2013.04.015

28. Park SY, Kwak YS. Impact of aerobic and anaerobic exercise training on oxidative stress and antioxidant defense in athletes. J Exerc Rehabil. 2016;12(2):113-117. doi:10.12965/ jer.1632598.299

29. Ezabadi A, Peeri M, Azarbayjani MA, Hosseini SA. The effects of resistance training and berberine chloride supplementation on oxidative stress markers in the cerebellum tissue of diazinon-poisoned rats. Middle East J Rehabil Health Stud. 2019;6(3):e92870. doi:10.5812/ mejrh. 92870

30. Kara M, Ozcagli E, Kotil T, Alpertunga B. Effects of stanozolol on apoptosis mechanisms and oxidative stress in rat cardiac tissue. Steroids. 2018;134:96-100. doi:10.1016/j. steroids.2018.02.004

31. Dousdampanis P, Trigka K, Fourtounas C, Bargman JM. Role of testosterone in the pathogenesis, progression, prognosis and comorbidity of men with chronic kidney disease. Ther Apher Dial. 2014;18(3):220-230. doi:10.1111/1744-9987.12101 